\title{
Pain control following inguinal herniorrhaphy: current perspectives
}

This article was published in the following Dove Press journal:

Journal of Pain Research

29 May 2014

Number of times this article has been viewed

\section{Martin F Bjurstrom ${ }^{1, *}$ \\ Andrea L Nicol ${ }^{2}$,* \\ Parviz K Amid ${ }^{3}$ \\ David C Chen ${ }^{3}$}

'Department of Anesthesiology, UCLA, Los Angeles, CA, USA;

${ }^{2}$ Department of Anesthesiology, University of Kansas, Kansas City, KS, USA; ${ }^{3}$ Department of Surgery, Lichtenstein Amid Hernia Clinic at UCLA, UCLA, Los Angeles, CA, USA

*These authors contributed equally to this work
Correspondence: David C Chen Lichtenstein Amid Hernia Clinic at UCLA, 1304 I5th Street, Suite 102, Santa Monica, CA 90404, USA

Tel + I 3103194080

Email dcchen@mednet.ucla.edu
Abstract: Inguinal hernia repair is one of the most common surgeries performed worldwide. With the success of modern hernia repair techniques, recurrence rates have significantly declined, with a lower incidence than the development of chronic postherniorrhaphy inguinal pain (CPIP). The avoidance of CPIP is arguably the most important clinical outcome and has the greatest impact on patient satisfaction, health care utilization, societal cost, and quality of life. The etiology of CPIP is multifactorial, with overlapping neuropathic and nociceptive components contributing to this complex syndrome. Treatment is often challenging, and no definitive treatment algorithm exists. Multidisciplinary management of this complex problem improves outcomes, as treatment must be individualized. Current medical, pharmacologic, interventional, and surgical management strategies are reviewed.

Keywords: inguinodynia, chronic postherniorrhaphy inguinal pain, inguinal hernia

\section{Introduction}

Inguinal hernia repair is one of the most common surgical procedures in the world. Annually, more than 20 million inguinal hernia repairs are conducted worldwide, and in the US alone the number reaches $800,000 .{ }^{1-4}$ In industrialized countries, lifetime risk for undergoing reparative surgery of inguinal hernia has been estimated to be $27 \%$ for men, and $3 \%$ for women. ${ }^{5}$ For a majority of patients, the operative correction of an inguinal hernia is successful with a minimum of postoperative sequelae, followed by a few weeks convalescence ${ }^{6}$ and no long-term complications. However, considering the vast number of patients undergoing inguinal hernia repair, any, even rare, severe long-term complication would be of great impact.

The techniques of operative repair, or herniorrhaphy, have been refined over the last decades and have resulted in open and laparoscopic tension-free approaches utilizing advanced prosthetic mesh-material as gold standard. Consequently, hernia recurrence rates have decreased dramatically $(1 \%-5 \%),{ }^{7}$ and today, chronic pain is instead recognized as the most significant complication. Chronic postsurgical pain (CPSP) has been defined as pain that develops after surgical intervention and lasts for at least 2 months, other causes of pain excluded. ${ }^{8} \mathrm{CPSP}$ has been historically reported with every common operation, including appendectomy, cholecystectomy, and cesarian section.

In the case of chronic postherniorrhaphy inguinal pain (CPIP), taking into account postoperative inflammatory processes, 3-6 months is usually the definition for chronicity. The reported frequency of chronic pain following inguinal hernia repair varies considerably between studies depending on differing definitions, end points, and methodologies, but the estimated risk of moderate to severe chronic pain 
is $10 \%-12 \% .^{9-11}$ The rate of chronic pain affecting activities of daily life or employment is agreed to be $0.5 \%-6.0 \%{ }^{4}$ which although being a conservative estimate constitutes an enormous individual and societal burden, translating to 4,000-48,000 new cases in the US annually, making it a health concern of tremendous scope.

The etiology and source of CPIP is complex and includes hernia recurrence, tissue inflammation, meshoma, and inguinal nerve injury or entrapment. ${ }^{12,13}$ Many patients suffer from neuropathic pain characterized by negative sensory phenomena, dysesthesia, allodynia, hyperalgesia, and nonneuropathic deep inflammatory pain. ${ }^{14}$ Peripheral and central sensitization, maladaptive neuronal plasticity, and neuroimmune alterations complicate the clinical picture and treatment of CPSP. ${ }^{15,16}$ Central sensitization, as it may relate to CPIP and other CPSP syndromes, has been shown in animal models to be modulated by NMDA (N-methyl-D-aspartate) and adenylate cyclase activation and upregulation following an inflammatory stimulus. This neuronal plasticity leads to the development of "pain vulnerability" or "hyperalgesic priming" which may represent a surrogate of pain chronicization in the transition from acute to chronic pain. ${ }^{17,18}$

CPIP is one of many significant pain syndromes that are related to surgical procedures. Mastectomies, thoracotomies, major abdominal surgeries, and lumbar spine surgery are all surgical procedures that have been shown to have higher rates of CPSP. ${ }^{19-21}$ Depending on the surgical procedure, the incidence of CPSP is $4 \%-50 \%,{ }^{19}$ thus indicating the importance of its prevention, management, and treatment.

Severe chronic pain is a devastating condition, arguably constituting its own neurological disease entity. ${ }^{22}$ Suffering is caused through painful symptoms, but also from associated affective disorders such as anxiety and depression, cognitive impairment, and somatic comorbidities. ${ }^{22,23}$ Sleep deprivation is a common consequence of chronic pain, contributing to worsening of the pain, ${ }^{22,24}$ and CPIP-patients have an overall significantly reduced quality of life. ${ }^{25}$ The exact socioeconomic burden of patients suffering from CPIP has not been calculated, but the total annual direct and indirect cost of a diagnosis related to chronic pain may range somewhere between US $\$ 9,000^{26}$ and US\$40,000 in the case of severe postsurgical neuropathic pain. ${ }^{27}$

Considering how common, detrimental, and costly CPIP is, prevention and skilled treatment of this condition is of utmost importance. This review will explain the multifactorial etiologies underlying CPIP, outline risk factors, describe symptomatology and evaluation, and most importantly, provide evidence-based concepts on current and prospective pharmacological, interventional, and surgical treatments of CPIP.

\section{Methods}

This is a comprehensive review of the literature from 1966 through October 2013, including reports, systematic reviews, scientific studies, and other literature concerning chronic pain following inguinal herniorrhaphy. The data were collected using the PubMed search engine (National Center for Biotechnology Information, US National Library of Medicine, USA), Cochrane Reviews, and a manual search of all pertinent references in the literature. The database searches were performed in September-October 2013, employing the keywords chronic, persistent, inguinal, groin, herniorrhaphy, hernioplasty, hernia repair, pain, and treatment in various combinations and also combined with specific treatment modalities. Relevant ongoing trials were searched using national and international trial registers (http://clinicaltrials.gov, https://www.clinicaltrialsregister. $\underline{\mathrm{eu}}$ ), and main investigators were contacted for further information. The language of publications and trials was not an exclusion criterion.

\section{Etiology}

The exact etiology of CPIP is complex and variable, with many patients exhibiting signs and symptoms that suggest multiple as opposed to singular etiologies. Classically, the differing etiologies can be explained by separating them into the differing types of pain encountered in CPIP: neuropathic, non-neuropathic, somatic, and visceral pain. The significant overlap and combinations of these types of pain makes diagnosis and treatment of CPIP a significant challenge.

Neuropathic pain in CPIP is thought to arise from damage or trauma to the inguinal nerves. The resultant pain usually develops in the sensory distribution of the affected nerve(s). The inguinal nerves that are involved in causing CPIP are the iliohypogastric nerve (IHN), ilioinguinal nerve (IIN), the genital branch of the genitofemoral nerve (GFN), and more rarely, the femoral branch of the GFN or the lateral femoral cutaneous nerve. Damage to these nerves can occur intraoperatively or postoperatively. Intraoperatively, nerves can be damaged by surgical manipulation, but also by stretching, crushing, electrical/thermal damage, partial or complete transection, becoming entrapped in suture during an open repair, or entrapment in tacks, suture, or fixation used during a laparoscopic repair. Postoperatively, nerves can become damaged due to envelopment within 
a meshoma, irritation secondary to an excessive fibrotic reaction, or inflammatory processes such as granuloma or neuroma formation. ${ }^{10,28}$

Causes of non-neuropathic pain include hernia recurrence, excessive scar tissue formation, pain from the bulk of the mesh, meshoma formation, ${ }^{12}$ or mesh-related excess fibrosis. ${ }^{29} \mathrm{~A}$ well-described CPIP pain syndrome that falls under the designation of somatic pain is periostitis pubis, most commonly due to deeply placed anchoring or periosteal anchoring of the mesh near the pubic tubercle. ${ }^{10,28,30,31}$ Visceral pain may arise from intestinal involvement with recurrence, incarceration, or mesh adherence or may be related to the spermatic cord (funiculodynia) or other periurethral structures, including venous congestion of the spermatic cord, dyssynergia of the ejaculatory effector muscles, stricture of the spermatic duct, or twisting of the spermatic cord. ${ }^{10,28,31}$ Centralization of pain and hyperalgesic priming is known to happen with any inflammatory insult, and this sensitization can last months after trauma or surgery, even if the patient has minimal reported pain. ${ }^{19,20}$ The inflammatory response to surgery may lead to centralization of pain through microglial activation and peripheral nociceptor activity predisposing toward "chronification of pain". ${ }^{17}$ Homeostasis is provided through endogenous opioid and mu-opioid receptor constituent activity. However, stress in the postoperative period can lead to a disruption of this system, thus leading to latent reactivation of hyperalgesia and, theoretically, to the transition from acute to chronic pain. ${ }^{19,20}$

\section{Risk factors}

Several preoperative, perioperative, and postoperative risk factors for the development of CPSP have been identified. ${ }^{1}$ All have not been thoroughly studied for CPIP, but an increasing number of studies support several of the listed factors (Table 1). In particular, two reviews examining publications on CPIP up until 2004,,${ }^{9,10}$ data from nationwide hernia databases in Scandinavian countries, ${ }^{3,35,36}$ Scotland, ${ }^{24}$ recent systematic reviews and meta-analyses, ${ }^{11,37-41}$ and a thorough prospective study by Aasvang et $\mathrm{al}^{42}$ provide substantial information. Table 1 shows known risk factors for CPIP.

Young age and female sex are two independent demographic risk factors, consistently identified in numerous large studies..$^{3,5,9,26,41}$ Women also seem to report more severe acute postoperative pain in general, ${ }^{46}$ which might be related to estrogen modulation of nociceptive processing. ${ }^{47}$ Although obesity has sometimes been denominated a risk factor, no firm conclusions can be made. ${ }^{9}$
Table I Risk factors for chronic postherniorrhaphy inguinal pain

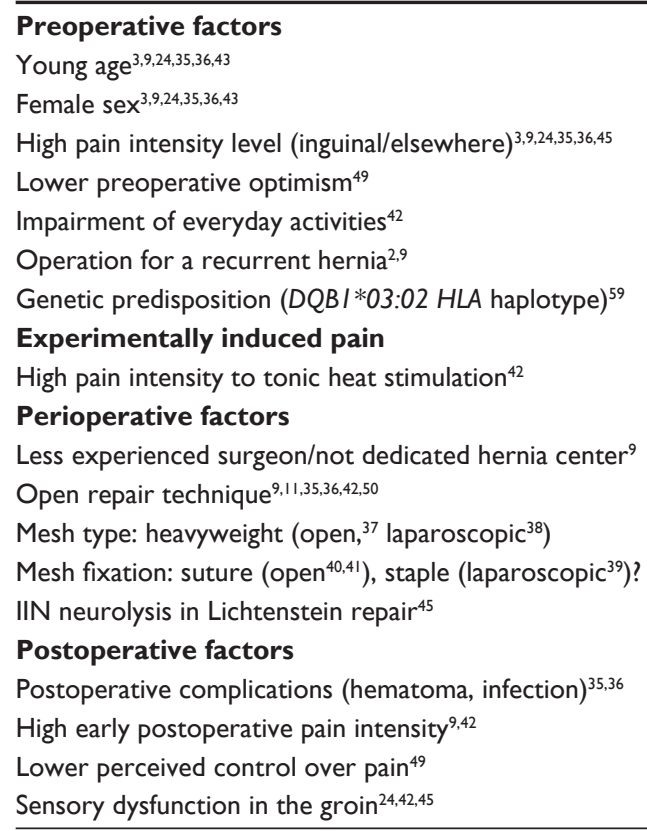

Note: ? = conflicting opinions/mixed evidence.

Abbreviations: IIN, ilioinguinal nerve; HLA, human leukocyte antigen.

Solid evidence underlie psychological and social factors influencing the development of CPSP (depression, psychological vulnerability, stress, and late return to work), ${ }^{48}$ but in the case of CPIP, thorough research in this field has not been conducted. In a small prospective cohort study with a short follow-up period, two cognitive (lower preoperative optimism and lower perceived control over pain) but no significant emotional risk factors for CPIP were found. ${ }^{49}$

High pre- and postoperative pain levels are strong risk factors for CPIP. ${ }^{3,9,24,35,36,45}$ In fact, a high magnitude of pain predicts future chronic pain risk consistently throughout the field of CPSP, and several mechanisms have been proposed, including perioperative nerve damage, sensitization of nociceptors in the surgical field, early postoperative ectopic activity of injured primary afferents, central sensitization, and structural changes in the central nervous system. ${ }^{32}$

The development of CPIP is independent of technique, and identification and protection of the inguinal nerves are of upmost importance with all techniques. ${ }^{4}$ Laparoscopic approaches may result in less chronic pain. ${ }^{9,11,35,36,42,50}$ However, the incidence of significant pain equilibrates over time, and pain after laparoscopic repair remains a significant challenge due to positioning of the mesh and proximal injury to the inguinal nerves. ${ }^{4,12,46}$ Mesh has early on been implicated as a culprit in the development of CPIP potentially causing both non-neuropathic (meshoma and fibrosis) and neuropathic (nerve entrapment) pain - hence 
various mesh materials and ways of fixating them have been examined.

Recent systematic reviews and meta-analyses have demonstrated significant reduction of CPIP for lightweight mesh compared with heavyweight mesh in both open and laparoscopic settings. ${ }^{37,38}$ The mechanism through which lightweight mesh reduces CPIP is thought to be greater biocompatibility (less inflammatory response) and elasticity similar to that of the abdominal wall, reducing stiffness and foreign body sensations. Several studies have addressed mesh fixation alternatives. At present, while results are mixed, it is a reasonable assertion that avoidance of sutures and tacks may reduce the incidence of CPIP. In one meta-analysis, glue fixation of mesh in open repair was shown to reduce CPIP, hematoma, acute postoperative pain, and time to return to daily activities. ${ }^{40}$ However, in another meta-analysis, only the latter and early CPIP (3-6 months) were significantly affected. ${ }^{41}$ In two other systematic reviews, it was concluded that glue mesh fixation is an interesting alternative, but that there is currently insufficient evidence regarding several end points due to the low quality of included studies. ${ }^{51,52}$ Based upon current evidence, self-gripping and sutured mesh demonstrate similar CPIP rates. ${ }^{53}$

Type of anesthesia - general, regional, or local - has not been extensively researched in connection to CPIP and presently cannot be linked to any significant CPIP outcome differences. ${ }^{36}$ However, it is not recommended to utilize regional anesthesia (epidural, spinal) for hernia repair, especially among older patients, due to an increased risk of urinary retention and other rare, but severe, medical complications. ${ }^{54}$ Local infiltration anesthesia is the preferred method for open repair, advantages including early recovery and discharge, less complications, improved early pain relief, and reduced costs. ${ }^{55-57}$ Results from a clinical trial studying the effect of perioperative infiltration of local anesthesia on the development of CPIP are pending (NCT00484731).

An interesting avenue for improving stratification of patients into low and high risk groups of developing CPIP is through preoperative experimental stimuli. Results from response to pain tests may predict $4 \%-54 \%$ of the variance in postoperative pain experience. ${ }^{58}$ Preoperative testing along with risk stratification may help to target at-risk patients who may benefit from implementation of specific preventive analgesic measures.

Finally, evidence from genetic research indicates an important role of an individual's genetic susceptibility - to both generation and experience of pain, and response to analgesics - in various chronic pain states. ${ }^{16,32}$ Functional genetic polymor- phisms have been identified in several genes (eg, COMT [encoding catecholamine-O-methyltransferase], $M C 1 R$ [encoding the melanocortin-1 receptor], 5-HTTLPR [encoding the serotonin transporter], $I L 1 B$ [encoding interleukin $\{$ IL $\}-1 \beta$ ], and ILIRN [encoding IL-1 receptor antagonist]), and linked directly or indirectly to different pain conditions. ${ }^{32}$ Dominguez et $\mathrm{al}^{59}$ recently demonstrated that the $D Q B 1 * 03: 02$ HLA (human leukocyte antigen) haplotype is associated with an increased risk of CPIP, and an ongoing multicenter Spanish study is studying the role of specific genes in the context of CPIP (NCT01510496).

\section{Symptomatology}

The etiologies contributing to CPIP are varied and complex in nature. Consequently, the symptomatology of CPIP is just as complex and variable and is dependent upon the type(s) of pain that the patient is suffering from: neuropathic pain, non-neuropathic pain, somatic pain, and/or visceral pain. Patients with neuropathic pain may describe having pain (neuralgia), reduced sensation (hypoesthesia), increased sensation (hyperesthesia), or a burning sensation (paresthesia). Occasionally, patients with neuropathic pain will report pain to a non-painful stimulus (allodynia) or increased pain response to a painful stimulus (hyperalgesia). Stabbing, burning, pulling, throbbing, shooting, prickling, and sharp are descriptors that are commonly used by patients suffering from neuropathic pain. ${ }^{30,60}$ These symptoms can be constant or intermittent. Pain can be localized or radiating in nature, with common sites of radiating pain being to the scrotum, labium, and/or upper thigh. ${ }^{31}$ Occasionally, a "trigger point" will exist and when palpated will cause the patient to experience the neuropathic pain symptoms. Neuropathic pain symptoms typically worsen with ambulation, twisting, or stretching of the upper body, stooping or sitting, hyperextension of the hip, or sexual intercourse and are made better with lying down or flexion of the hip and thigh. ${ }^{10,28,31}$

Non-neuropathic pain in CPIP is typically described as a dull ache, typically constant, usually over the entirety of the groin area, with no specific trigger point or radiating component. ${ }^{28,31}$ Words typically used to describe this type of pain include: gnawing, tender, pounding, or pulling. Somatic pain, as discussed previously, is usually localized to the pubic tubercle area as the area of maximum tenderness. Visceral pain in CPIP is generally related to sexual dysfunction or ejaculatory pain in the region of the superficial ring or the testicular/labial region. According to a study performed by Loos et al, ${ }^{31}$ patients with CPIP visceral pain symptoms describe them as aching, without a specific trigger point. 
Those with a history of anterior approach who have visceral pain have a spermatic cord that is diffusely tender to palpation. ${ }^{31}$

When evaluating a patient with chronic groin pain after hernia surgery, it is important to take into consideration the breadth of differential diagnoses (surgical, orthopedic, neurologic, infectious disease, urologic, or gynecologic), despite the inclination to suspect CPIP as the most logical cause. Hernia recurrence is a source of chronic pain in this population, is relatively easy to evaluate, and should be ruled out early in the evaluation. ${ }^{61}$

\section{Evaluation}

The evaluation of a patient with CPIP should characterize the cause and type of pain and exclude differential diagnostic entities. A detailed history and clinical examination is essential. Administration of validated pain, function and quality-of-life instruments such as the Short Form McGill Pain Questionnaire (SF-MPQ), Neuropathic Pain Questionnaire (NPQ), or Douleur Neuropathique 4 Questions (DN4), SF-36 (36-Item Short Form Health Survey), and Activity Assessment Scale are recommended. The screening tools NPQ and DN4 have high specificity for neuropathic pain, and if a neuropathic component is identified, it can be further characterized using the NPS (Neuropathic Pain Scale) or SF-MPQ-2. ${ }^{62}$

Tinel's test (tapping the skin medial to the anterior superior iliac spine or over the area of maximum tenderness) can reproduce neuropathic pain distributed along the sensory innervation of the affected nerve(s). Based on these findings, neuropathic, non-neuropathic, or mixed pain can be diagnosed. Due to overlapping sensory innervations, peripheral communication between IHN, IIN, and GFN nerve twigs and common routes of origin, it is very difficult to ascertain which nerves are actually involved in the neuralgic pain. ${ }^{63-65}$ Ultrasonography is a common initial modality to detect recurrence or meshoma. ${ }^{66}$ Cross-sectional computed tomography (CT) or magnetic resonance imaging (MRI) of the abdominal wall may exclude these pathologies and other differential diagnoses of CPIP. ${ }^{61}$ Currently, MRI is considered the best valid diagnostic imaging tool for differentiating causes of uncertain inguinal pain, ${ }^{67}$ but results and interpretation is radiologist dependent. ${ }^{68}$ Diagnostic peripheral nerve block or paravertebral root block with a local anesthetic may help to identify ilioinguinal, iliohypogastric, and/or genitofemoral neuralgia. Typically no further guidance is offered through other evaluation tools, but when results of nerve blocks are equivocal, needle electromyogram might provide additional information (eg, through revealing abnormalities and denervation of the abdominal muscles specific for IHN/IIN-injury). ${ }^{69,70}$ Magnetic resonance neurography may identify peripheral nerve compression that will improve with surgical treatment but is also technique and radiologist dependent. ${ }^{71}$

\section{Treatment Multidisciplinary non-interventional pain management}

CPIP is complex. It is not only a product of neuropathic pain and nociceptive components but is also influenced and modulated by emotional, cognitive, social, and genetic factors. A multimodal, multidisciplinary treatment approach is therefore necessary. Neuropathic pain is difficult to treat, and the pain is complicated by central sensitization and psychological comorbidities. Lacking firm specific CPIPevidence-based non-interventional treatment strategies, the best conclusions we can currently draw are from small studies on CPIP, empiric evidence, and extrapolation of evidence from other neuropathic and CPSP conditions.

\section{Non-pharmacological treatment}

The perception of pain depends on multiple factors such as experience, fears, context, and meaning and can be modulated through cognition and emotion. Physiotherapy, acupuncture, and mind-body therapies provide ways of ameliorating pain conditions and are important cornerstones in a multimodal approach. ${ }^{72}$ No specific studies address these therapies in CPIP, but an array of mind-body therapies have been shown to improve recovery time and pain following surgery and childbirth, for example, and also in other chronic pain conditions. ${ }^{73}$ Certain lifestyle measures such as recumbent position or flexion of the hip and thigh might provide temporary relief of painful symptoms; it is, however, important to avoid harmful inactivity.

\section{Pharmacological treatment}

In case of deep, inflammatory non-neuropathic pain, and even when neuropathic pain is caused by nerve entrapment secondary to inflammation and edema, non-steroidal antiinflammatory drugs (NSAIDs) and steroids can be used with some degree of success. It is, however, rarely sustainable to continue with these medications long-term due to side effects. There are no head-to-head comparisons between basic analgesics (eg, NSAIDs, steroids, and acetaminophen) in the context of CPIP.

Recent guidelines on pharmacological treatment of neuropathic pain (International Association for the Study of 
Pain [IASP $],{ }^{74}$ European Federation of Neurological Societies $\left[\right.$ EFNS], ${ }^{75,76}$ and Canadian Pain Society ${ }^{77}$ ) systematically analyze the evidence of various treatment options based on randomized clinical trials. Although most available trials have investigated neuropathic pain in the context of postherpetic neuralgia (PHN) or painful diabetic peripheral neuropathy, and follow-up has usually been relatively short ( $\leq 3$ months), it is reasonable to extrapolate results between neuropathic conditions. $^{78}$

Presently, it is not possible to rank the pharmacological alternatives for the individual patient. It is important to choose treatment according to factors including potential side effects, concomitant treatment of other comorbidities (anxiety, depression, and insomnia), drug interactions, risk of abuse, and cost. There are some discrepancies between the above-stated guidelines, but all taken together and adjusted for the case of CPIP, it is reasonable to start with either calcium channel $\alpha_{2}-\delta$ ligands (gabapentin and pregabalin) or antidepressants with both norepinephrine and serotonin reuptake inhibition (SSNRIs) (tricyclic antidepressants [TCAs]).

Gabapentin and pregabalin are structural analogs of GABA ( $\gamma$-Aminobutyric acid). Titration over a few weeks is needed, and most side effects are transient and dose dependent. Dizziness and sedation are commonly observed. It is hypothesized that $\alpha_{2}-\delta$ ligands given preoperatively at a certain dosage and for an adequate duration might be able to diminish postoperative pain and prevent establishment of central sensitization; and meta-analysis supports this idea. ${ }^{79}$ Unfortunately a trial designed to investigate the effect of pregabalin in CPIP was stopped prematurely due to low inclusion rate (NCT00772291). There is a general scarcity of trials examining pharmacological alternatives in CPIP. ${ }^{80}$ In a small suboptimally designed study (utilizing spinal anesthesia and lacking comprehensive pain measures), gabapentin administered preoperatively to inguinal hernia repair patients was shown to significantly decrease pain scores at 6 months follow-up - an interesting finding in relation to potential prevention of CPIP. ${ }^{81}$ Considering the difficulties in treating CPIP there has been an increasing focus on preventive analgesia; however, a study on tumor necrosis factor- $\alpha$ inhibitor etanercept administered preoperatively failed to show beneficial long-term results. ${ }^{82}$

Duloxetine and venlafaxine are SSNRIs ranked as firstline alternatives by IASP, but second-line by EFNS and the Canadian Pain Society. Advantages of TCAs are low cost, easy dosing, and beneficial effect on anxiety, depression, and sleep; while disadvantages are risk for anticholinergic side effects and orthostatic hypotension. There is a risk for cardiotoxicity, and due to an association between TCAs at dosages $\geq 100 \mathrm{mg}$ and sudden death, caution should be exercised in patients with a history of ischemic heart disease and dosages preferentially held $\leq 100 \mathrm{mg}$.

Opioids and tramadol are considered second-line treatment alternatives for neuropathic pain but can be utilized as first-line during episodic exacerbations of severe neuropathic pain or during titration of TCAs, SSNRIs, or $\alpha_{2}-\delta$ ligands. Although opioids provide NNTs (numbers needed to treat) equal to TCAs $(2-3)^{83}$ in various neuropathic pain conditions, side-effects and concerns related to long-term treatment (immunological, hormonal, opioid-induced hyperalgesia, abuse, and dependence) limit their long-term use. Few alternatives can provide as swift pain relief as opioids, and they are often a necessary component of the treatment armamentarium. The most common side effects are constipation, nausea, and sedation; prophylactic bowel regimen and antiemetic must be considered and add to the cost of treatment. There is often a need for combination therapy, and the strongest evidence supports TCA-gabapentin or gabapentin-opioids. ${ }^{75}$

In the case of failure of, or contraindication to, first- and second-line treatment alternatives, a few other medications can be considered, although their evidence in neuropathic pain is meager or conflicting. SSRIs (selective serotonin reuptake inhibitors) (citalopram and paroxetine), bupropion, cannabinoids, anticonvulsants (lamotrigine, oxcarbazepine, topiramate, and valproic acid), or dextromethorphan, memantine, clonidine, and mexiletine may be tried, based upon patient characteristics and specific circumstances.

\section{Topical medications}

Considering the relatively limited anatomical area causing pain in CPIP, it is reasonable to attempt a trial of topical medications. However, these alternatives may not absorb far enough to treat the underlying condition, and at present, no firm supportive evidence for lidocaine and capsaicin patches exists for CPIP. Lidocaine acts on voltage-gated sodium channels on hyperactive or damaged nociceptors, decreasing afferent nociceptive input, and has been shown effective in $\mathrm{PHN}$ and in various types of peripheral neuropathic pain with allodynia. ${ }^{84,85}$ However, in a well-designed small crossover trial $(n=21)$ of lidocaine patches $(5 \%)$ in patients with severe CPIP, no reduction of pain ratings compared with placebo were found. ${ }^{86}$

Capsaicin's analgesic effect is exerted through repeated applications causing persistent desensitization. While awaiting 
results from a Danish ongoing study examining capsaicin patches (8\%) in CPIP (NCT01699854), evidence of capsaicin's effect in neuropathic pain has to be derived from other pain conditions. In a Cochrane review from 2013, with results based on PHN and HIV (human immunodeficiency virus)neuropathy, NNTs for at least $30 \%$ pain intensity reduction week 2-12 were ten and eleven, respectively. ${ }^{87}$ Based on the high cost of repeated applications, scarce evidence and likely high NNTs, topical medications may be tried as second-line treatment or when the patient's comorbidities complicate use of the first-line alternatives.

\section{Interventional pain management}

Overall, there is a paucity of evidence-based medicine evaluating the safety and efficacy of interventional pain management techniques in the treatment of CPIP. Few welldesigned, randomized, prospective research studies in this field have been performed. Most of the available information regarding these techniques exists in the form of case reports or case series. In the following sections, the most commonly employed interventional pain management techniques and their respective pertinent available literature and evidencebased medicine will be reported and discussed.

\section{Nerve blocks}

Nerve blocks of the IIN, IHN, and/or GFN have been used for both diagnostic and therapeutic purposes in the diagnosis and treatment of CPIP. In some studies, they have been used preoperatively to determine which patients may potentially respond favorably to surgical neurectomy. Historically, these nerve blocks were performed using a blind technique, using anatomic landmarks as guidance for needle placement. While a blind technique for IIN and IHN blocks would still allow for administration of the local anesthetic proximal to the site of injury, there exists no blind technique that would allow for this with the GFN, given its anatomic course.

Recent advances in ultrasound technology and its use in regional anesthesia have enabled practitioners to perform IIN and IHN blocks under a direct visualization, with successful outcomes reducing risk of intraperitoneal needle placement and allowing for a smaller volume of injectate due to improved accuracy. While there has been a published case report on ultrasound-guided GFN block, the target for needle placement was approached lateral to the pubic tubercle, which may be too distal when considering a nerve injury or entrapment after open groin hernia repair. ${ }^{88,89}$ Ultrasound-guided techniques for selective block of the GFN proximal to the site of nerve injury are not currently described or available. ${ }^{89}$
A recent case report describing a CT-guided trans-psoas GFN block appears to be a promising technique to safely block the GFN proximal to the site of nerve injury. ${ }^{90}$

Few studies have been performed evaluating the efficacy of ultrasound-guided IIN/IHN blocks in the treatment of CPIP, and provide conflicting results. ${ }^{91,92}$ In a recent report by Thomassen et al, ${ }^{92}$ the authors found ultrasoundguided IIN/IHN blocks to be effective in treating CPIP with successful prolonged pain relief after a median follow-up time of 20 months. Despite this finding, they reported that they did not find any statistically significant difference in pain outcomes when they compared patients who received nervestimulator-guided nerve blocks versus ultrasound-guided nerve blocks. Only one randomized, double-blind, placebocontrolled study has been published to date evaluating the efficacy of ultrasound-guided IIN/IHN blocks in the treatment of CPIP. ${ }^{89}$ Two cohorts of patients (CPIP and healthy control groups) were studied in this crossover design trial. The results of this study by Bischoff et $\mathrm{al}^{89}$ failed to provide substantial evidence to support the use of ultrasound-guided IIN/IHN blocks in the diagnosis and treatment of CPIP. A study comparing the use of ultrasound-guided IIN/IHN blocks to operative neurectomy is currently underway. ${ }^{93}$

\section{Neurolysis/neuroablative techniques}

If nerve blocks have been performed and provided significant analgesia but did not provide long-term relief, neuroablative techniques such as chemical neurolysis, cryoablation, and pulsed radiofrequency (PRF) ablation may be considered for longer-lasting effect. While chemical neurolysis using phenol or alcohol has been used in the treatment of CPIP, ${ }^{90,94}$ most reports on neuroablative techniques include those using cryoablation or PRF ablation techniques.

Cryoablation is neurodestructive by means of Wallerian degeneration and selectively destroys axons and myelin sheaths while leaving the epineurium and perineurium intact. The affected axons treated with cryoablation are very unlikely to form neuromas, and patients are less likely to develop deafferentation pain, both of which have been associated with neurectomy or thermal non-PRF ablation. Cryoablation has been reported as being a successful mode of analgesia for patients with CPIP. Fanelli et $\mathrm{al}^{95}$ reported the use of this modality in nine patients, where they performed cryoablation of the nerves under direct surgical visualization, and the mean overall pain reduction was $77.5 \%$. Campos et $\mathrm{al}^{96}$ published the first reported cryoablation of the femoral branch of the GFN under ultrasound guidance in a patient with chronic inguinal pain; however, this patient did not suffer from 
CPIP, and thus the applicability of this procedure to the CPIP population is unclear.

The use of PRF ablation in the treatment of CPIP has been reported in multiple case series ${ }^{97-100}$ as well as in a recent systematic review. ${ }^{101} \mathrm{PRF}$ ablation delivers high intensity currents in pulses, which allows for heat (typically $42^{\circ} \mathrm{C}$ ) to dissipate during the latent phase so that neurodestructive temperatures are not obtained, thus lowering the risk of neuroma formation, neuritistype reaction, and deafferentation pain. This mild heating of the nerve tissue is thought to temporarily block nerve conduction; however, the exact mechanism by which PRF ablation provides analgesia is unclear. Rozen and $\mathrm{Ahn}^{97}$ and Rozen and Parvez ${ }^{98}$ described in two published case series (of the same patient cohort) the use of PRF ablation of the T12, L1, and L2 lumbar nerve roots for treatment of CPIP. In these repeat publications, the five patients had $75 \%-100 \%$ pain reduction that lasted 6-9 months, leading the authors to conclude that this modality is a potentially efficacious treatment option for patients suffering from CPIP. In the published case series by Cohen and Foster, ${ }^{99}$ three patients with chronic groin pain (two of them were CPIP) underwent either IIN and/or IHN or GFN PRF ablation after having successful diagnostic blocks. Compared with the Rozen and Ahn, and Rozen and Parvez studies, ${ }^{97,98}$ the PRF ablation in the study by Cohen and Foster, ${ }^{99}$ was performed peripherally based on anatomic location of these nerves in the groin, and correct needle placement was confirmed using sensory stimulation. All three of these patients reported continued complete pain relief at 6 months. Mitra et al ${ }^{100}$ published a single case report on the use of PRF ablation for a patient with non-CPIP ilioinguinal neuralgia. Similarly to Cohen and Foster, ${ }^{99}$ they report performing PRF ablation peripherally at the anatomic location of the nerves in the groin, using sensory stimulation as confirmation for correct needle placement. In this study, they describe a significant reduction in pain score (from VAS [visual analog scale] 8/10 to 3/10) that persisted at a 3-month follow-up..$^{100}$

Werner et al ${ }^{101}$ recently published a systematic review of the available data on PRF ablation in the treatment of CPIP. They report that there is a limited level of evidence to support the use of PRF ablation in the management of CPIP, stating that the evidence is of low quality and the strength of recommendation is weak to moderate. They propose improved scientific rigor and requirements for further PRF ablation studies to fully examine the safety and efficacy of this interventional technique.

\section{Neuromodulation}

For some patients, their CPIP will be refractory to pharmacologic, interventional, and possibly even surgical treatment. Neuromodulation techniques, either peripheral nerve field stimulation (PNFS) or spinal cord stimulation (SCS), may be considered for use in a select group of patients when all other conventional treatments have failed. Historically, the gate control theory of pain was used to describe the mechanism by which SCS and PNFS provided analgesia; however, currently, the exact neurophysiologic mechanisms of action for both of these modalities is not yet completely understood. ${ }^{102,103}$ PNFS and SCS are implantable devices that provide pain relief by producing gentle paresthesias in the concordant areas of pain. Multiple case reports or case series of either separate PNFS, ${ }^{104-109}$ SCS, ${ }^{110-112}$ or combined PNFS and SCS ${ }^{113}$ have been published, all with compelling and successful results. Patient selection appears to play a large role in the success of these modalities. A few authors have recommended a stringent and selective screening process that advocates for the selection of well-motivated and relatively young patients who are not involved in litigation, have success with a pre-implantation trial period, have favorable spinal anatomy (which would improve easier placement of SCS compared with those with unfavorable anatomy or prior spine surgery), and pass a preimplantation psychiatric evaluation. ${ }^{105,112}$ Further large-scale studies may allow for improved evidence and strength of recommendation for these two promising modalities.

\section{Surgical pain management}

While the majority of patients with CPIP can be managed with pharmacologic, interventional, and behavioral measures, those refractory to a systematic regimen of diagnostic and interventional measures may be considered for operative remediation. Successful outcomes, however, are entirely dependent upon choosing patients with discrete, neuroanatomic problems that may be corrected with surgery. ${ }^{1,4,114,115}$ Failure of conservative measures, in and of itself, is not an indication for further surgery. There is no level 1 or 2 evidence regarding the operative management of inguinodynia, and best available recommendations are derived from case reports, case series, expert opinion, and expert consensus. ${ }^{4}$ Development of inguinodynia is independent of the method of hernia repair, ${ }^{3,4114}$ but an in-depth understanding of the causes of pain, groin neuroanatomy, and technical aspects of the initial operation are necessary to successfully manage these patients. ${ }^{1,4,12,114,116-118}$ These factors determine the operative options available to address chronic pain after inguinal hernia repair.

The neuroanatomy of the groin is complex and highly variable from the retroperitoneal lumbar plexus to the terminal 
branches exiting through the inguinal canal. Understanding the location of potential nerve injury is crucial. ${ }^{64,119}$ In front of transversalis fascia, the IIN, the visible and intramuscular segment of the IHN, and the inguinal segment of the genital branch of the GFN must be considered. These structures may potentially be injured during open anterior repairs (tissue repair, Lichtenstein, PHS [prolene hernia system], and plug) and from mesh fixation during laparoscopic repair (TEP [totally extraperitoneal] and TAPP [transabdominal preperitoneal]). Behind the transversalis fascia within the preperitoneal space, the main trunk of the genitofemoral and the preperitoneal segment of genital branch of GFN are at risk. These must be considered during open preperitoneal repair (plug, PHS, and Kugel) and laparoscopic repair (TEP and TAPP). Finally, injury to the nerves within the retroperitoneal space including the main trunk of the GFN over the psoas muscle and the lateral femoral cutaneous nerve must also be considered after open and laparoscopic posterior repair. ${ }^{18,120}$

Recommended timing for surgical treatment of chronic postherniorrhaphy pain not responding to nonsurgical management is 6 months to 1 year after the original hernia repair. ${ }^{1,4}$ A systematic and thorough preoperative evaluation to identify the specific etiologies of pain is imperative. This assessment should include symptomatology, review of the prior operative report for technique (specifically, type of repair, type of mesh used, position of the mesh, method of fixation, and nerve handling), imaging to assess for meshoma or other anatomic abnormality, and response with prior interventions. ${ }^{4,13,30}$ Neuropathic pain isolated to the inguinal distribution, that was not present prior to the original operation, and with improvement from diagnostic and therapeutic nerve blocks has the highest likelihood of improvement with operative neurectomy.

Operative management for pain after inguinal surgery has been reported as early as 1942 with Magee ${ }^{121}$ describing genitofemoral causalgia as a source for post-inguinal surgery pain. Selective IIN, IHN, and GFN neurolysis or neurectomy, removal of mesh and fixation material, and revision of the prior herniorrhaphy are common options for treatment. ${ }^{122-127}$ Neurolysis, which does not address ultrastructural changes of nerve fibers, has limited efficacy and is not recommended. ${ }^{4}$ Simple removal of entrapping sutures or fixating devices while leaving the injured nerves behind is also inadequate. ${ }^{4}$ Selective single or double neurectomy may be effective for some patients but does not address ultrastructural changes of seemingly normal appearing nerves during reoperation. ${ }^{122-125}$ From a technical perspective, reoperating in the scarred field becomes increasingly more difficult and morbid for subsequent remedial operations. Anatomically, the significant variation and cross-innervation of the inguinal nerves in the retroperitoneum and inguinal canal make selective neurectomy less reliable. ${ }^{4,64,119}$ Triple neurectomy of the IIN, IHN, and GFN, described in our institute in 1995, is currently a universally accepted surgical treatment for neuropathic pain refractory to conservative measures and is arguably the most effective option. ${ }^{4,12,64,116-120}$ Our experience has included over 650 patients utilizing an open approach with an over $85 \%$ success rate and 37 cases using a laparoscopic retroperitoneal approach with a $92 \%$ success rate. ${ }^{120}$ Operative neurectomy in conjunction with removal of meshoma, when present, provides effective relief in the majority of patients with refractory inguinodynia., $4,119,120$

"Triple neurectomy" can be performed through an open approach using the groin incision of the original hernia operation or through a laparoscopic approach particularly for pain after plug repair and open and laparoscopic preperitoneal repair. ${ }^{114}$ With open surgery, the IIN can be identified lateral to the internal ring, between the ring and the anterior superior iliac spine. The IHN is identified within the anatomic cleavage between the external and internal oblique aponeurosis. The nerve is then traced proximally within the fibers of the internal oblique muscle to a point lateral to the field of the original hernia repair. Failure to do so may leave the injured intramuscular segment of the nerve behind. In those instances where the IHN has a subaponeurotic course, the internal oblique aponeurosis is split to visualize and address the hidden nerve. The inguinal segment of the genital branch of the GFN can be identified between the cord and the inguinal ligament and traced laterally to the internal ring where it is severed. Alternatively, the nerve may be visualized within the internal ring through the lateral crus of the ring. The nerves should be resected proximal to the field of original hernia repair. Although there are no specific data available, we recommend ligation of the cut ends of the nerves to avoid neuroma formation and insertion of the proximal cut end into the muscle to keep the nerve stump away from the future scarring of the operative field..$^{4116-118}$ Advantages of the open approach are the possibility of performing a single stage operation for triple neurectomy as well as plug/meshoma removal if any, repair of the resulting defect, resection of the main trunk of the GFN over psoas muscle, and resection of paravasal nerves within the lamina propria of the vas in case of associated orchialgia. The disadvantage of the approach is its complexity and technical difficulty operating within the scarred field, 
placing the spermatic cord and vascular structures at higher risk of compromise.

Laparoscopic retroperitoneal triple neurectomy may be performed through a transabdominal or extraperitoneal approach. ${ }^{120,128,129}$ The IIN and IHN can be identified within the retroperitoneal space over the quadratus lumborum muscle and the GFN over the psoas muscle proximal to the scarred operative field. Endoscopic retroperitoneal selective neurectomies of the GFN and IIN have been reported. ${ }^{128,129}$ As with the open experience, laparoscopic retroperitoneal triple neurectomy has higher efficacy rates. ${ }^{120}$ Advantages of the laparoscopic approach are the ability to access the nerves proximal to the mesh material used during the original herniorrhaphy, more consistent neuroanatomy within the retroperitoneum, and its technical simplicity. Disadvantages of laparoscopic approach are not being able to remove plugs, if any, not being able to resect the lamina propria of the vas in case of associated orchialgia, and potential laxity of the abdominal muscles caused from proximal denervation.

It is critical to clearly explain potential benefits and consequences of operative intervention to manage patient expectations. In addition to the usual operative risks, specific considerations include permanent numbness, the inability to access or identify three nerves, abdominal wall laxity from partial denervation of the oblique muscles, testicular atrophy, numbness in the labia in females that can interfere with sexual sensation, and loss of a cremasteric reflex in male patients. ${ }^{4,118-120}$ Patients are specifically advised of the potential for ongoing pain and disability despite successful neurectomy due to the nociceptive component of pain, neuroplasticity, afferent hypersensitivity, and centralization of pain. The development and course of deafferentation hypersensitivity is unpredictable but typically diminishes over time. This operation does not address the nociceptive component caused by meshoma or neuropathic testicular pain. These issues require serious consideration and should be discussed with the patient and adequately recorded. ${ }^{4}$

Recurrence and meshoma are obvious anatomic pathologies amenable to surgical correction. When recurrence is identified, surgical correction is typically recommended using an alternative approach (ie, laparoscopic repair after initial open repair or vice versa). However, if accompanied by neuropathic pain, an anterior, open approach allows for correction of the hernia as well as access to the nerves. ${ }^{118}$ Meshoma may cause neuropathic pain from nerve entrapment, direct contact with mesh, or compressive effects. ${ }^{13,29}$ It may also cause nociceptive pain from compression of adjacent structures and foreign body sensation. Imaging is useful to help identify meshoma and involvement of adjacent structures. Operative removal of the meshoma is indicated with the need for simultaneous neurectomy directed by the type of mesh, approach, symptoms, imaging, and anatomy. If coexisting neuropathic pain is present, all nerves within the reoperative field should be addressed, as neuropathy cannot be assessed visually, and mesh removal will often compromise unaffected nerves within the inguinal canal. ${ }^{4}$

Neuropathy of the nerve fibers within the lamina propria of the vas deferens, referred to as "paravasal nerves" and originating from the deep pelvic plexus, may be partially responsible for postherniorrhaphy orchialgia. In patients with groin pain associated with orchialgia, segmental resection of the lamina propria of the vas together with triple neurectomy has improved outcomes and helped in the management of testicular pain. ${ }^{118}$ Orchialgia, however, is a complex entity, and remedial surgery to correct this is less predictable and effective.

\section{Conclusion}

Chronic inguinodynia is a problem for which there is no universally accepted definition, etiology, classification, and surgical treatment. In-depth knowledge of groin neuroanatomy is critical, as the best measure to address this disabling complication is prevention by refining the technique of hernia repair. Meticulous adherence to surgical principles, with three-nerve identification, preservation, or pragmatic neurectomy during open anterior repair, decreases the incidence of chronic inguinodynia. Avoidance of the preperitoneal nerves below the iliopubic tract and limited or no mesh fixation decreases the risk of pain after laparoscopic herniorrhaphy.

Patients at high risk of developing CPIP should be identified preoperatively, and preventive multimodal analgesia and anesthesia should be given high priority. Preventive measures such as peri- and postoperative gabapentinoids or ketamine treatment, or infiltration of local anesthetic, might minimize the inflammatory cascade, decrease central sensitization, and prevent development of severe chronic pain. The recommended timing for surgical treatment of chronic postherniorrhaphy pain not responding to nonsurgical management is 6 months to 1 year after the original inguinal hernia repair. A systematic and thorough evaluation to identify the potential causes of pain is mandatory. This assessment should include symptoms, review of the prior operative report for technique, imaging to assess for meshoma or other anatomic abnormalities, and thorough conservative management with pharmacologic and interventional modalities. 
All patients with CPIP should undergo multidisciplinary treatment with evaluation by a pain specialist. Pharmacologic intervention including anti-inflammatories, topical anesthetics, neuropathic medications, antidepressants, and opioid analgesics should be optimized. All patients should undergo diagnostic and therapeutic nerve block of the IIN, IHN, and GFN as appropriate. Nerve ablation and neuromodulation are promising interventional modalities that may help in the non-operative management of CPIP and may serve as adjunctive therapy for patients who have failed reoperative surgery or with centralization of pain. For patients with pain refractory to conservative measures, operative neurectomy, meshoma removal, and repair of recurrence may provide relief. A multidisciplinary, logical, stepwise approach to chronic postoperative inguinal pain will afford patients the greatest opportunity to minimize symptoms, manage pain, decrease further morbidity, and improve quality of life.

\section{Disclosure}

There are no relevant disclosures or conflicts of interest reported by any of the authors.

\section{References}

1. Aasvang E, Kehlet H. Surgical management of chronic pain after inguinal hernia repair. Br J Surg. 2005;92:795-801.

2. Aasvang EK, Bay-Nielsen M, Kehlet H. Pain and functional impairment 6 years after inguinal herniorrhaphy. Hernia. 2006;10:316-321.

3. Bay-Nielsen M, Perkins FM, Kehlet H. Pain and functional impairment 1 year after inguinal herniorrhaphy: a nationwide questionnaire study. Ann Surg. 2001;233:1-7.

4. Alfieri S, Amid PK, Campanelli G, et al. International guidelines for prevention and management of post-operative chronic pain following inguinal hernia surgery. Hernia. 2011;15:239-249.

5. Primatesta P, Goldacre MJ. Inguinal hernia repair: incidence of elective and emergency surgery, readmission and mortality. Int J Epidemiol. 1996;25:835-839.

6. Bay-Nielsen M, Thomsen H, Andersen FH, et al. Convalescence after inguinal herniorrhaphy. Br J Surg. 2004;91:362-367.

7. Bittner R, Schwarz J. Inguinal hernia repair: current surgical techniques. Langenbecks Arch Surg. 2012;397:271-282.

8. Macrae WA, Davies HTO. Chronic postsurgical pain. In: Crombie IK, Croft PR, Linton SJ, LeResche L, Von Korff M, editors. Epidemiology of Pain. Seattle: IASP Press; 1999: 125-142.

9. Aasvang E, Kehlet H. Chronic postoperative pain: the case of inguinal herniorrhaphy. Br J Anaesth. 2005;95:69-76.

10. Poobalan AS, Bruce J, Smith WC, King PM, Krukowski ZH, Chambers WA. A review of chronic pain after inguinal herniorrhaphy. Clin J Pain. 2003;19:48-54.

11. Nienhuijs S, Staal E, Strobbe L, Rosman C, Groenewoud H, Bleichrodt R. Chronic pain after mesh repair of inguinal hernia: a systematic review. Am J Surg. 2007;194:394-400.

12. Amid PK, Hiatt JR. New understanding of the causes and surgical treatment of postherniorrhaphy inguinodynia and orchalgia. $\mathrm{JAm}$ Coll Surg. 2007;205:381-385.

13. Amid PK. Radiologic images of meshoma: a new phenomenon causing chronic pain after prosthetic repair of abdominal wall hernias. Arch Surg. 2004;139:1297-1298.
14. Haroutiunian S, Nikolajsen L, Finnerup NB, Jensen TS. The neuropathic component in persistent postsurgical pain: a systematic literature review. Pain. 2013;154:95-102.

15. Woolf CJ. Central sensitization: implications for the diagnosis and treatment of pain. Pain. 2011;152:S2-S15.

16. Kehlet H, Jensen TS, Woolf CJ. Persistent postsurgical pain: risk factors and prevention. Lancet. 2006;367:1618-1625.

17. Rivat C, Laboureyras E, Laulin JP, Le Roy C, Richebé P, Simonnet G. Nonnociceptive environmental stress induces hyperalgesia, not analgesia, in pain and opioid-experienced rats. Neuropsychopharmacol. 2007;32:2217-2228.

18. Corder G, Doolen S, Donahue RR, et al. Constitutive $\mu$-opioid receptor activity leads to long-term endogenous analgesia and dependence. Science. 2013;341:1394-1399.

19. Rivat C, Bollag L, Richebé P. Mechanisms of regional anaesthesia protection against hyperalgesia and pain chronicization. Curr Opin Anaesthesiol. 2013;26:621-625.

20. Eisenach JC. Preventing chronic pain after surgery: who, how and when? Reg Anesth Pain Med. 2006;31(1):1-3.

21. Chan CW, Peng P. Failed back surgery syndrome. Pain Med. 2011;12(4):577-606.

22. Fine PG. Long-term consequences of chronic pain: mounting evidence for pain as a neurological disease and parallels with other chronic disease states. Pain Med. 2011;12:996-1004.

23. Tsang A, Von Korff M, Lee S, et al. Common chronic pain conditions in developed and developing countries: gender and age differences and comorbidity with depression-anxiety disorders. J Pain. 2008;9: 883-891.

24. Courtney CA, Duffy K, Serpell MG, O'Dwyer PJ. Outcome of patients with severe chronic pain following repair of groin hernia. Br J Surg. 2002;89:1310-1314.

25. Kalliomaki ML, Sandblom G, Gunnarsson U, Gordh T. Persistent pain after groin hernia surgery: a qualitative analysis of pain and its consequences for quality of life. Acta Anaesthesiol Scand. 2009;53:236-246.

26. Gustavsson A, Bjorkman J, Ljungcrantz C, et al. Socio-economic burden of patients with a diagnosis related to chronic pain - register data of 840,000 Swedish patients. Eur J Pain. 2012;16:289-299.

27. Parsons B, Schaefer C, Mann R, et al. Economic and humanistic burden of post-trauma and post-surgical neuropathic pain among adults in the United States. J Pain Res. 2013;6:459-469.

28. Hakeem A, Shanmugam V. Current trends in the diagnosis and management of post-herniorraphy chronic groin pain. World $J$ Gastrointest Surg. 2011;3:73-81.

29. Heise CP, Starling JR. Mesh inguinodynia: a new clinical syndrome after inguinal herniorrhaphy? J Am Coll Surg. 1998;187:514-518.

30. Cunningham J, Temple WJ, Mitchell P, Nixon JA, Preshaw RM, Hagen NA. Cooperative hernia study. Pain in the postrepair patient. Ann Surg. 1996;224:598-602.

31. Loos MJ, Roumen RM, Scheltinga MR. Classifying post-herniorrhaphy pain syndromes following elective inguinal hernia repair. World J Surg. 2007;31:1760-1765; discussion 1766-1767.

32. Katz J, Seltzer Z. Transition from acute to chronic postsurgical pain: risk factors and protective factors. Expert Rev Neurother. 2009;9:723-744.

33. Perkins FM, Kehlet H. Chronic pain as an outcome of surgery. A review of predictive factors. Anesthesiology. 2000;93:1123-1133.

34. VanDenKerkhof EG, Peters ML, Bruce J. Chronic pain after surgery: time for standardization? A framework to establish core risk factor and outcome domains for epidemiological studies. Clin J Pain. 2013;29:2-8.

35. Franneby U, Sandblom G, Nordin P, Nyren O, Gunnarsson U. Risk factors for long-term pain after hernia surgery. Ann Surg. 2006;244: 212-219.

36. Kalliomaki ML, Meyerson J, Gunnarsson U, Gordh T, Sandblom G. Long-term pain after inguinal hernia repair in a population-based cohort; risk factors and interference with daily activities. Eur J Pain. 2008;12:214-225. 
37. Sajid MS, Leaver C, Baig MK, Sains P. Systematic review and metaanalysis of the use of lightweight versus heavyweight mesh in open inguinal hernia repair. Br J Surg. 2012;99:29-37.

38. Sajid MS, Kalra L, Parampalli U, Sains PS, Baig MK. A systematic review and meta-analysis evaluating the effectiveness of lightweight mesh against heavyweight mesh in influencing the incidence of chronic groin pain following laparoscopic inguinal hernia repair. Am J Surg. 2013;205:726-736.

39. Kaul A, Hutfless S, Le H, et al. Staple versus fibrin glue fixation in laparoscopic total extraperitoneal repair of inguinal hernia: a systematic review and meta-analysis. Surg Endosc. 2012;26:1269-1278.

40. Colvin HS, Rao A, Cavali M, Campanelli G, Amin AI. Glue versus suture fixation of mesh during open repair of inguinal hernias: a systematic review and meta-analysis. World J Surg. 2013;37:2282-2292.

41. de Goede B, Klitsie PJ, van Kempen BJ, et al. Meta-analysis of glue versus sutured mesh fixation for Lichtenstein inguinal hernia repair. Br J Surg. 2013;100:735-742.

42. Aasvang EK, Gmaehle E, Hansen JB, et al. Predictive risk factors for persistent postherniotomy pain. Anesthesiology. 2010;112: 957-969.

43. Bay-Nielsen M, Nilsson E, Nordin P, Kehlet H. Chronic pain after open mesh and sutured repair of indirect inguinal hernia in young males. Br J Surg. 2004;91:1372-1376.

44. Sondenaa K, Nesvik I, Breivik K, Korner H. Long-term follow-up of 1059 consecutive primary and recurrent inguinal hernias in a teaching hospital. Eur J Surg. 2001;167:125-129.

45. Reinpold WM, Nehls J, Eggert A. Nerve management and chronic pain after open inguinal hernia repair: a prospective two phase study. Ann Surg. 2011;254:163-168.

46. Kalkman CJ, Visser K, Moen J, Bonsel GJ, Grobbee DE, Moons KG. Preoperative prediction of severe postoperative pain. Pain. 2003;105: 415-423.

47. Amandusson A, Blomqvist A. Estrogenic influences in pain processing. Front Neuroendocrinol. 2013;34:329-349.

48. Hinrichs-Rocker A, Schulz K, Järvinen I, Lefering R, Simanski C, Neugebauer EA. Psychosocial predictors and correlates for chronic post-surgical pain (CPSP) - a systematic review. Eur J Pain. 2009;13: 719-730.

49. Powell R, Johnston M, Smith WC, et al. Psychological risk factors for chronic post-surgical pain after inguinal hernia repair surgery: a prospective cohort study. Eur J Pain. 2012;16:600-610.

50. Hindmarsh AC, Cheong E, Lewis MP, Rhodes M. Attendance at a pain clinic with severe chronic pain after open and laparoscopic inguinal hernia repairs. Br J Surg. 2003;90:1152-1154.

51. Ladwa N, Sajid MS, Sains P, Baig MK. Suture mesh fixation versus glue mesh fixation in open inguinal hernia repair: a systematic review and meta-analysis. Int J Surg. 2013;11:128-135.

52. Sanders DL, Waydia S. A systematic review of randomised control trials assessing mesh fixation in open inguinal hernia repair. Hernia. 2014;18(2):165-176.

53. Zhang C, Li F, Zhang H, Zhong W, Shi D, Zhao Y. Self-gripping versus sutured mesh for inguinal hernia repair: a systematic review and meta-analysis of current literature. J Surg Res. 2013;185(2): 653-660.

54. Bay-Nielsen M, Kehlet H. Anaesthesia and post-operative morbidity after elective groin hernia repair: a nation-wide study. Acta Anaesthesiol Scand. 2008;52:169-174.

55. Kehlet H, Aasvang E. Groin hernia repair: anesthesia. World J Surg. 2005;29:1058-1061.

56. Nordin P, Zetterstrom H, Carlsson P, Nilsson E. Cost-effectiveness analysis of local, regional and general anaesthesia for inguinal hernia repair using data from a randomized clinical trial. Br J Surg. 2007;94: $500-505$.

57. Nordin P, Zetterstrom H, Gunnarsson U, Nilsson E. Local, regional, or general anaesthesia in groin hernia repair: multicentre randomised trial. Lancet. 2003;362:853-858.
58. Werner MU, Mjobo HN, Nielsen PR, Rudin A. Prediction of postoperative pain: a systematic review of predictive experimental pain studies. Anesthesiology. 2010;112:1494-1502.

59. Dominguez CA, Kalliomäki M, Gunnarsson U, et al. The DQB1 * 03:02 HLA haplotype is associated with increased risk of chronic pain after inguinal hernia surgery and lumbar disc herniation. Pain. 2013;154:427-433.

60. Choi PD, Nath R, Mackinnon SE. Iatrogenic injury to the ilioinguinal and iliohypogastric nerves in the groin: a case report, diagnosis, and management. Ann Plast Surg. 1996;37:60-65.

61. Ferzli GS, Edwards ED, Khoury GE. Chronic pain after inguinal herniorrhaphy. J Am Coll Surg. 2007;205:333-341.

62. Rab M, Ebmer And J, Dellon AL. Anatomic variability of the ilioinguinal and genitofemoral nerve: implications for the treatment of groin pain. Plast Reconstr Surg. 2001;108:1618-1623.

63. Jones RC 3rd, Backonja MM. Review of neuropathic pain screening and assessment tools. Curr Pain Headache Rep. 2013;17(9):363.

64. Starling JR, Harms BA. Diagnosis and treatment of genitofemoral and ilioinguinal neuralgia. World J Surg. 1989;13:586-591.

65. Harms BA, DeHaas DR Jr, Starling JR. Diagnosis and management of genitofemoral neuralgia. Arch Surg. 1984;119:339-341.

66. Bradley M, Morgan D, Pentlow B, Roe A. The groin hernia - an ultrasound diagnosis? Ann R Coll Surg Engl. 2003;85:178-180.

67. van den Berg JC, de Valois JC, Go PM, Rosenbusch G. Detection of groin hernia with physical examination, ultrasound, and MRI compared with laparoscopic findings. Invest Radiol. 1999;34:739-743.

68. Aasvang EK, Jensen KE, Fiirgaard B, Kehlet H. MRI and pathology in persistent postherniotomy pain. J Am Coll Surg. 2009;208:1023-1028; discussion 1028-1029.

69. Ellis RJ, Ceisse H, Holub BA. Ilioinguinal nerve conduction. Muscle Nerve. 1992;15:1195.

70. Knockaert DC, Boonen AL, Bruyninckx FL, Bobbaers HJ. Electromyographic findings in ilioinguinal-iliohypogastric nerve entrapment syndrome. Acta Clin Belg. 1996;51:156-160.

71. Filler A. Magnetic resonance neurography and diffusion tensor imaging: origins, history, and clinical impact of the first 50,000 cases with an assessment of efficacy and utility in a prospective 5000-patient study group. Neurosurgery. 2009;65:A29-A43.

72. Bushnell MC, Ceko M, Low LA. Cognitive and emotional control of pain and its disruption in chronic pain. Nat Rev Neurosci. 2013;14: 502-511.

73. Astin JA. Mind-body therapies for the management of pain. Clin J Pain. 2004;20:27-32.

74. Dworkin RH, O'Connor AB, Backonja M, et al. Pharmacologic management of neuropathic pain: evidence-based recommendations. Pain. 2007;132:237-251.

75. Attal N, Cruccu G, Baron R, et al. EFNS guidelines on the pharmacological treatment of neuropathic pain: 2010 revision. Eur J Neurol. 2010;17:1113. e88.

76. Attal N, Cruccu G, Haanpää M, et al. EFNS guidelines on pharmacological treatment of neuropathic pain. Eur J Neurol. 2006;13:1153-1169.

77. Moulin DE, Clark AJ, Gilron I, et al. Pharmacological management of chronic neuropathic pain - consensus statement and guidelines from the Canadian Pain Society. Pain Res Manag. 2007;12:13-21.

78. O'Connor AB, Dworkin RH. Treatment of neuropathic pain: an overview of recent guidelines. Am J Med. 2009;122:S22-S32.

79. Clarke H, Bonin RP, Orser BA, Englesakis M, Wijeysundera DN, Katz J. The prevention of chronic postsurgical pain using gabapentin and pregabalin: a combined systematic review and meta-analysis. Anesth Analg. 2012;115:428-442.

80. Benito-Leon J, Picardo A, Garrido A, Cuberes R. Gabapentin therapy for genitofemoral and ilioinguinal neuralgia. J Neurol. 2001;248: 907-908.

81. Sen H, Sizlan A, Yanarateş O, et al. The effects of gabapentin on acute and chronic pain after inguinal herniorrhaphy. Eur J Anaesthesiol. 2009;26:772-776. 
82. Cohen SP, Galvagno SM, Plunkett A, et al. A multicenter, randomized, controlled study evaluating preventive etanercept on postoperative pain after inguinal hernia repair. Anesth Analg. 2013;116:455-462.

83. Finnerup NB, Otto M, McQuay HJ, Jensen TS, Sindrup SH. Algorithm for neuropathic pain treatment: an evidence based proposal. Pain. 2005;118:289-305

84. Garnock-Jones KP, Keating GM. Lidocaine 5\% medicated plaster: a review of its use in postherpetic neuralgia. Drugs. 2009;69: 2149-2165.

85. Mick G, Correa-Illanes G. Topical pain management with the 5\% lidocaine medicated plaster - a review. Curr Med Res Opin. 2012;28:937-951.

86. Bischoff JM, Petersen M, Uçeyler N, Sommer C, Kehlet H, Werner MU. Lidocaine patch $(5 \%)$ in treatment of persistent inguinal postherniorrhaphy pain: a randomized, double-blind, placebocontrolled, crossover trial. Anesthesiology. 2013;119(6):1444-1452.

87. Derry S, Sven-Rice A, Cole P, Tan T, Moore RA. Topical capsaicin (high concentration) for chronic neuropathic pain in adults. Cochrane Database Syst Rev. 2013;2:CD007393.

88. Peng PW, Tumber PS. Ultrasound-guided interventional procedures for patients with chronic pelvic pain - a description of techniques and review of literature. Pain Physician. 2008;11:215-224.

89. Bischoff JM, Koscielniak-Nielsen ZJ, Kehlet H, Werner MU. Ultrasound-guided ilioinguinal/iliohypogastric nerve blocks for persistent inguinal postherniorrhaphy pain: a randomized, doubleblind, placebo-controlled, crossover trial. Anesth Analg. 2012;114: 1323-1329.

90. Parris D, Fischbein N, Mackey S, Carroll I. A novel CT-guided transpsoas approach to diagnostic genitofemoral nerve block and ablation. Pain Med. 2010;11:785-789.

91. Gofeld M, Christakis M. Sonographically guided ilioinguinal nerve block. J Ultrasound Med. 2006;25:1571-1575.

92. Thomassen I, van Suijlekom JA, van de GaagA, Ponten JE, Nienhuijs SW. Ultrasound-guided ilioinguinal/iliohypogastric nerve blocks for chronic pain after inguinal hernia repair. Hernia. 2013;17:329-332.

93. Loos MJ, Verhagen T, Scheltinga MR, Roumen RM. A randomised controlled trial of injection therapy versus neurectomy for post-herniorrhaphy inguinal neuralgia: rationale and study design. Hernia. 2010;14:593-597.

94. Aasvang EK, Brandsborg B, Christensen B, Jensen TS, Kehlet H. Neurophysiological characterization of postherniotomy pain. Pain. 2008;137:173-181.

95. Fanelli RD, DiSiena MR, Lui FY, Gersin KS. Cryoanalgesic ablation for the treatment of chronic postherniorrhaphy neuropathic pain. Surg Endosc. 2003;17:196-200.

96. Campos NA, Chiles JH, Plunkett AR. Ultrasound-guided cryoablation of genitofemoral nerve for chronic inguinal pain. Pain Physician. 2009;12:997-1000.

97. Rozen D, Ahn J. Pulsed radiofrequency for the treatment of ilioinguinal neuralgia after inguinal herniorrhaphy. Mt Sinai J Med. 2006;73:716-718.

98. Rozen D, Parvez U. Pulsed radiofrequency of lumbar nerve roots for treatment of chronic inguinal herniorraphy pain. Pain Physician. 2006;9:153-156.

99. Cohen SP, Foster A. Pulsed radiofrequency as a treatment for groin pain and orchialgia. Urology. 2003;61:645.

100. Mitra R, Zeighami A, Mackey S. Pulsed radiofrequency for the treatment of chronic ilioinguinal neuropathy. Hernia. 2007;11:369-371.

101. Werner MU, Bischoff JM, Rathmell JP, Kehlet H. Pulsed radiofrequency in the treatment of persistent pain after inguinal herniotomy: a systematic review. Reg Anesth Pain Med. 2012;37: 340-343.

102. Wall PD, Sweet WH. Temporary abolition of pain in man. Science. 1967;155:108-109.

103. Melzack R, Wall PD. Pain mechanisms: a new theory. Science. 1965;150:971-979.
104. Rosendal F, Moir L, de Pennington N, Green AL, Aziz TZ. Successful treatment of testicular pain with peripheral nerve stimulation of the cutaneous branch of the ilioinguinal and genital branch of the genitofemoral nerves. Neuromodulation. 2013;16:121-124.

105. Banh DP, Moujan PM, Haque Q, Han TH. Permanent implantation of peripheral nerve stimulator for combat injury-related ilioinguinal neuralgia. Pain Physician. 2013;16:E789-E791.

106. Rauchwerger JJ, Giordano J, Rozen D, Kent JL, Greenspan J, Closson CW. On the therapeutic viability of peripheral nerve stimulation for ilioinguinal neuralgia: putative mechanisms and possible utility. Pain Pract. 2008;8:138-143.

107. Stinson LW Jr, Roderer GT, Cross NE, Davis BE. Peripheral subcutaneous electrostimulation for control of intractable post-operative inguinal pain: a case report series. Neuromodulation. 2001;4:99-104

108. Paicius RM, Bernstein CA, Lempert-Cohen C. Peripheral nerve field stimulation in chronic abdominal pain. Pain Physician. 2006;9: 261-266.

109. Carayannopoulos A, Beasley R, Sites B. Facilitation of percutaneous trial lead placement with ultrasound guidance for peripheral nerve stimulation trial of ilioinguinal neuralgia: a technical note. Neuromodulation. 2009;12:296-301.

110. Alo KM, Yland MJ, Redko V, Feler C, Naumann C. Lumbar and sacral nerve root stimulation (NRS) in the treatment of chronic pain: a novel anatomic approach and neuro stimulation technique. Neuromodulation 1999;2:23-31.

111. Elias M. Spinal cord stimulation for post-herniorrhaphy pain. Neuromodulation. 2000;3:155-157.

112. Yakovlev AE, Al Tamimi M, Barolat G, et al. Spinal cord stimulation as alternative treatment for chronic post-herniorrhaphy pain. Neuromodulation. 2010;13:288-290; discussion 291.

113. Mironer YE, Monroe TR. Spinal-peripheral neurostimulation (SPN) for bilateral postherniorrhaphy pain: a case report. Neuromodulation. 2013;16(6):603-606.

114. Lichtenstein IL, Shulman AG, Amid PK, Montllor MM. Cause and prevention of postherniorrhaphy neuralgia: a proposed protocol for treatment. Am J Surg. 1988;155(6):786-790.

115. Kehlet H. Chronic pain after groin hernia repair. Br J Surg. 2008;95: 135-136.

116. Amid PK. A 1-stage surgical treatment for postherniorrhaphy neuropathic pain: triple neurectomy and proximal end implantation without mobilization of the cord. Arch Surg. 2002;137(1): 100-104.

117. Amid PK. Causes, prevention, and surgical treatment of postherniorrhaphy neuropathic inguinodynia: triple neurectomy with proximal end implantation. Hernia. 2004;8(4):343-349.

118. Amid PK, Chen DC. Surgical treatment of chronic groin and testicular pain after laparoscopic and open preperitoneal inguinal hernia repair. J Am Coll Surg. 2011;213(4):531-536.

119. Klaassen Z, Marshall E, Tubbs RS, Louis RG Jr, Wartmann CT, Loukas M. Anatomy of the ilioinguinal and iliohypogastric nerves with observations of their spinal nerve contributions. Clin Anat. 2011;24(4):454-461

120. Chen DC, Hiatt JR, Amid PK. Operative management of refractory neuropathic inguinodynia by a laparoscopic retroperitoneal approach JAMA Surg. 2013;148(10):962-967.

121. Magee RK. Genitofemoral causalgia: (a new syndrome). Can Med Assoc J. 1942;46(4):326-329.

122. Aasvang EK, Kehlet $H$. The effect of mesh removal and selective neurectomy on persistent postherniotomy pain. Ann Surg. 2009;249(2): 327-334.

123. Starling JR, Harms BA. Ilioinguinal, iliohypogastric, and genitofemoral neuralgia. In: Bendavid R, editor. Prostheses and Abdominal Wall Hernia. Austin, TX: RG Landes Co; 1994:351-356.

124. Zacest AC, Magill ST, Anderson VC, Burchiel KJ. Long-term outcome following ilioinguinal neurectomy for chronic pain. J Neurosurg. 2010;112(4):784-789. 
125. Loos MJ, Scheltinga MR, Roumen RM. Tailored neurectomy for treatment of postherniorrhaphy inguinal neuralgia. Surgery. 2010;147(2):275-281.

126. Madura JA, Madura JA 2nd, Copper CM, Worth RM. Inguinal neurectomy for inguinal nerve entrapment: an experience with 100 patients. Am J Surg. 2005;189(3):283-287.

127. Keller JE, Stefanidis D, Dolce CJ, Iannitti DA, Kercher KW, Heniford BT. Combined open and laparoscopic approach to chronic pain after inguinal hernia repair. Am Surg. 2008;74:695-700.
128. Song JW, Wolf JS Jr, McGillicuddy JE, Bhangoo S, Yang LJ. Laparoscopic triple neurectomy for intractable groin pain: technical report of 3 cases. Neurosurgery. 2011;68(2):339-346; discussion 346.

129. Giger U, Wente $\mathrm{MN}$, Büchler MW, Krähenbühl S, Lerut J, Krähenbühl L. Endoscopic retroperitoneal neurectomy for chronic pain after groin surgery. Br J Surg. 2009;96(9):1076-1081.

\section{Publish your work in this journal}

The Journal of Pain Research is an international, peer-reviewed, open access, online journal that welcomes laboratory and clinical findings in the fields of pain research and the prevention and management of pain. Original research, reviews, symposium reports, hypothesis formation and commentaries are all considered for publication.

\section{Dovepress}

The manuscript management system is completely online and includes a very quick and fair peer-review system, which is all easy to use. Visit http://www.dovepress.com/testimonials.php to read real quotes from published authors. 